\title{
Research on fault diagnosis of rolling bearings based on conditional variance statistic and cross-correlation spectrum
}

\author{
Baoyu Huang ${ }^{1}$, Yongxiang Zhang ${ }^{2}$, Danchen $\mathrm{Zhu}^{3}$ \\ ${ }^{1,2}$ Department of Power Engineering, Naval University of Engineering, Wuhan, China \\ ${ }^{3}$ Department of Mechanical and Electrical Engineering, Naval Petty Officer Academy, Bengbu, China \\ ${ }^{2}$ Corresponding author \\ E-mail:12632643625@qq.com, 22yx6302@aliyun.com,3bryantzdc@sina.com
}

Received 18 September 2020; received in revised form 4 December 2020; accepted 17 December 2020 DOI https://doi.org/10.21595/jve.2020.21698

Check for updates

Copyright $(2021$ Baoyu Huang, et al. This is an open access article distributed under the Creative Commons Attribution License, which permits unrestricted use, distribution, and reproduction in any medium, provided the original work is properly cited.

\begin{abstract}
In this paper, relationships between conditional variance statistic and the center frequency as well as the bandwidth of the filter are researched, and we find that conditional variance statistic is suitable for selecting the center frequency of the filter, but undesirable for selecting the bandwidth. Meanwhile, considering that the traditional iterative way to select the optimal resonance frequency band will be affected by the step size, we choose to fix the bandwidth at five times fault characteristic frequency, then Whale Optimization Algorithm (WOA) is utilized to select the optimal center frequency so as to give consideration to both computing efficiency and accuracy of the selection of center frequency. To further suppress the in-band noise, the filtered signal is analyzed by high order energy operator, and the optimal two energy operators are chosen based on Fault Characteristic Index (FCI) for cross-correlation spectrum analysis. Simulation and experimental results indicate that the proposed algorithm can extract the fault feature of rolling bearings under strong background noise effectively.
\end{abstract}

Keywords: fault diagnosis, conditional variance statistic, cross-correlation spectrum, rolling bearing.

\section{Introduction}

Rolling bearings are widely used as one of the important parts of rotating machinery, and their operating conditions directly affect the performance of the whole machine. Therefore, condition monitoring and fault diagnosis for rolling bearings are particularly important. Due to the complexity of mechanical equipment and its harsh operating environment, bearing fault signals are often submerged in strong background noise, and the measured vibration signals are usually non-stationary and nonlinear, which greatly increase the difficulty of fault feature extraction of bearing vibration signals.

In order to extract the fault feature of rolling bearings under strong background noise, researchers have proposed many effective methods, such as wavelet transform [1], empirical mode decomposition (EMD) [2], variational mode decomposition (VMD) [3], blind deconvolution algorithm [4-7] and so on. However, strong background noise will interfere with the selection of wavelet basis, which directly affects the effectiveness of wavelet transform; EMD has the problems of end effect and mode mixing, and the algorithm lacks rigorous mathematical reasoning; the performance of VMD depends on the selection of decomposition number and penalty parameter seriously; minimum entropy deconvolution (MED), which aims to restore single dominant pulse, is susceptible to random impulse noise; what's more, the performance of blind deconvolution algorithm based on the cyclostationarity of fault signal, such as maximum correlated kurtosis deconvolution (MCKD), multipoint optimal minimum entropy deconvolution (MOMEDA) and maximum second-order cyclostationarity blind deconvolution (CYCBD) relies on the selection of fault period, which is usually difficult due to speed fluctuations or rolling element slippage. 
Envelope analysis is the most mature method in bearings' fault diagnosis [8], its performance depends on the correct selection of resonance frequency band. To solve the problem, Antoni proposed Fast Kurtogram to select the resonance frequency band based on the maximum kurtosis [9]. However, the center frequency and bandwidth selected by Fast Kurtogram are not accurate enough, which results in the loss of some useful information, especially under strong non-Gaussian noise, Fast Kurtogram usually fails to extract the fault feature [10]. To solve these problems, Xu et al. [11] constructed a set of filters with variable bandwidth by empirical wavelet transform and obtained a series of empirical modal components, then the optimal empirical mode component was selected according to the maximum kurtosis, simulation and experimental results verified the effectiveness of the method. Based on the cyclostationarity of bearing fault signal, Barszcz et al. [12] proposed protrugram, which fixed the bandwidth and selected the best filter parameters with the maximum squared envelope spectrum kurtosis. However, this method ignores the impulsiveness of the bearing fault signal, which may influence its performance. To alleviate this problem, Antoni took into account the impulsiveness of the fault signal in the time domain and the cyclostationarity in the frequency domain, then proposed infogram [13]. This algorithm calculated the negentropy of squared envelope signal and squared envelope spectrum, then their mean value was used as the objective function to select the optimal frequency band, simulation and experimental results verified its effectiveness. However, under strong impulsive noise, the negentropy of the squared envelope signal may be much larger than that of the squared envelope spectrum, which makes the selected filter band favors impulsiveness more than cyclostationarity [14]. Recently, Gini index which is a measure of sparsity has been used to extract the fault feature of rolling bearings. Miao et al. [15] found that Gini index is more robust to random impulse noise than kurtosis, and used it to select the resonance frequency band. Moreover, he further compared Gini index with kurtosis, results showed that although Gini index is more robust to impulsive noise than kurtosis, its robustness to random white noise is weaker [16].

Justyna et al. [14] performed a short-time Fourier transform on signal and selected the resonance frequency band based on the maximum conditional variance statistic, results showed the feasibility of this algorithm. Then he further compared conditional variance statistic with kurtosis, Gini index, smoothness index and stability index (Alpha selector), results indicated that if the fault signal didn't contain impulsive noise, all indicators could select the resonant frequency band appropriately, but the method based on the conditional variance statistic performed best; if the fault signal contained strong impulsive noise, only the method based on conditional variance statistic could accurately select the resonant frequency band of the signal [17]. However, the algorithm proposed by Justyna also has the following disadvantages: if the step size is too short, it will affect the calculation efficiency; if the step size is too long, the precise center frequency cannot be selected.

Aiming at the problem that it is difficult to extract the fault feature of rolling bearings under strong background noise, and considering the strong robustness of conditional variance statistic, we propose to optimize the parameters of the filter by conditional variance statistic. What's more, this paper further researches the effect of bandwidth and center frequency on conditional variance statistic, results show that it can be used as the objective function to select the center frequency but is unreliable to select bandwidth. Fortunately, Barszcz et al. [12] pointed out that bandwidth should be more than three times of fault characteristic frequency. Duan et al. [18] further pointed out that when the bandwidth is $n$ times of fault characteristic frequency, it may also fail to extract $n$ fault related harmonics in the squared envelope spectrum, and proposed that the bandwidth should be slightly greater than four times of fault characteristic frequency. Based on the above considerations, we fix the bandwidth at five times fault characteristic frequency, and choose conditional variance statistic as the objective function, then WOA is utilized to select the optimal center frequency quickly; in order to further suppress the in-band noise, the filtered signal is analyzed by high-order energy operator, and the optimal two energy operators are selected based on FCI for cross-correlation spectrum analysis; finally, the fault feature is extracted by fast Fourier transform. 


\section{Basic principles}

In this section, conditional variance statistic is researched, and results indicate that it can be used as the objective function for selecting the center frequency of the filter, but is not suitable for selecting the bandwidth. Hence, we fix the bandwidth at five times fault characteristic frequency, and WOA is utilized to quickly select the appropriate center frequency; then the filtered signal is analyzed by high-order energy operator analysis; finally, the optimal two energy operators are selected based on FCI for cross-correlation spectrum analysis.

\subsection{Selection of the optimal filtering frequency band}

\subsubsection{Conditional variance statistic}

Conditional variance statistic is derived from the statistical phenomenon commonly known as the 20/60/20 rule. Suppose a set of data $Y$ conforms to a Gaussian random distribution, with mean $\alpha$ and standard deviation $\sigma . \Phi_{\alpha, \sigma}$ denotes its distribution function. when $0<q<0.5$, we can obtain left partitioning $L_{q}$, right partitioning $R_{q}$ and middle partitioning $M_{q}$ of $Y$ using Eq. (1):

$$
\left\{\begin{array}{l}
L_{q}=\left(-\infty, \phi_{a, \sigma}^{-1}(q)\right], \\
R_{q}=\left[\phi_{a, \sigma}^{-1}(1-q), \infty\right), \\
M_{q}=\left(\phi_{a, \sigma}^{-1}(q), \phi_{a, \sigma}^{-1}(1-q)\right),
\end{array}\right.
$$

where $\phi_{a, \sigma}^{-1}(q)$ denotes the inverse of $\phi_{a, \sigma}$, if $Y$ conforms to normality assumption and $q$ equals to 0.2 , we get that:

$$
\sigma_{L_{q}}^{2}=\sigma_{R_{q}}^{2}=\sigma_{M_{q}}^{2}
$$

where $\sigma_{A}^{2}=\operatorname{Var}(Y \mid Y \in A)$ is the conditional variance of $Y$ on set $A$, and Eq. (2) is only satisfied when $q$ equals to 0.2 [19].

Due to the fact that $q=0.2$ is the unique value to satisfy Eq. (2), a goodness-of-fit test statistic can be constructed by it. In other words, we can determine whether the sample belongs to Gaussian distribution by the comparation on the value of conditional variance and the conditional central variance [14]. On this basis, literature [20] proposed a test statistic to measure the degree of heavy tail of the data. Given a set of data $Y=\left(Y_{1}, \ldots, Y_{n}\right)$, there is a statistical test:

$N=\frac{1}{\rho}\left(\frac{\hat{\sigma}_{L_{q}}^{2}-\hat{\sigma}_{M_{q}}^{2}}{\hat{\sigma}^{2}}+\frac{\hat{\sigma}_{R_{q}}^{2}-\hat{\sigma}_{M_{q}}^{2}}{\hat{\sigma}^{2}}\right) \sqrt{n}$,

where $q$ equals to $0.2, \rho \in R$ is a constant, $n$ is the length of the signal, $\hat{\sigma}^{2}$ denotes sample variance and $\hat{\sigma}_{A}^{2}$ is the conditional sample variance on set $A$.

Literature [20] pointed out that the asymptotic distribution of $N$ is standard normal if the sample is independent and identically distributed. Moreover, if the sample belongs to (symmetric) heavy tailed distribution, the values of statistic $N$ should be larger than 0 because of the high values of conditional tail variances on sets $L_{q}$ and $R_{q}$. Hence, $N$ could be utilized to measure the fatness of tail, and the bigger the value of $N$, the fatter the tails [14].

For seven quantile conditioning subsets, the (unique) ratio guaranteeing conditional variance equality is equal to 0:004/0:058/0:246/0:384/0:246/0:058/0:004, it can be expressed as: 


$$
\begin{aligned}
& \left\{\begin{array}{l}
A_{1}=\left(-\infty, \phi_{a, \sigma}^{-1}(0.004)\right], \\
A_{2}=\left(\phi_{a, \sigma}^{-1}(0.004), \phi_{a, \sigma}^{-1}(0.062)\right], \\
A_{3}=\left(\phi_{a, \sigma}^{-1}(0.062), \phi_{a, \sigma}^{-1}(0.308)\right], \\
A_{4}=\left(\phi_{a, \sigma}^{-1}(0.308), \phi_{a, \sigma}^{-1}(0.692)\right], \\
A_{5}=\left(\phi_{a, \sigma}^{-1}(0.692), \phi_{a, \sigma}^{-1}(0.938)\right], \\
A_{6}=\left(\phi_{a, \sigma}^{-1}(0.938), \phi_{a, \sigma}^{-1}(0.996)\right], \\
A_{7}=\left(\phi_{a, \sigma}^{-1}(0.996), \infty\right),
\end{array}\right. \\
& \sigma_{A_{1}}=\sigma_{A_{2}}=\sigma_{A_{3}}=\sigma_{A_{4}}=\sigma_{A_{5}}=\sigma_{A_{6}}=\sigma_{A_{7}} .
\end{aligned}
$$

Considering that extreme sample tail sets estimators might be related to noise components, only choose to construct test statistics with sample conditional variance of set $A_{3}, A_{4}$ and $A_{5}$, which can be expressed as [14]:

$N_{2}=\left(\frac{\hat{\sigma}_{A_{3}}^{2}-\hat{\sigma}_{A_{4}}^{2}}{\hat{\sigma}^{2}}+\frac{\hat{\sigma}_{A_{5}}^{2}-\hat{\sigma}_{A_{4}}^{2}}{\hat{\sigma}^{2}}\right)^{2} \sqrt{n}$,

where $\hat{\sigma}^{2}$ is the sample variance and $\hat{\sigma}_{A}^{2}$ is the conditional sample variance on $A, n$ is the length of the signal. In literature [14], Justyna used $N_{2}$ to measure the impact of the non-extreme (trimmed) tail variance on the central part of the distribution and squared the variance sum in Eq. (6) to get more accurate cluster split.

Justyna selected the resonant frequency band of the signal by $N_{2}$ [14], which can be explained as follow: when a faulty bearing is operating, it will produce resonance of the system. If the selected filter frequency band happens to be the resonant frequency band of the system, the filtered signal will definitely behave with heavy tail distribution, the value of $N_{2}$ will be larger; else, the filtered signal will be closer to the Gaussian distribution and $N_{2}$ will be closer to 0 .

However, our research find that $N_{2}$ can be used as an objective function for selecting the center frequency of the filter, but it is not suitable for selecting the bandwidth. To prove these findings, this paper constructs a faulty simulation signal as Eq. (7):

$$
\left\{\begin{array}{l}
x_{1}(t)=\sum_{i=1}^{N} A_{i} s\left(t-i T-t_{i}\right)+B(t)+n(t)+m(t) \\
A_{i}=4 \cos \left(2 \pi f_{r} t+\frac{\pi}{2}\right)+0.5 \\
B(t)=1.2 \cos \left(2 \pi f_{1} t+\frac{\pi}{2}\right)+0.9 \cos \left(2 \pi f_{2} t\right) \\
s(t)=e^{-2 \pi f_{n} r t} \sin \left(2 \pi f_{n} t\right)
\end{array}\right.
$$

where, $f_{r}=42 \mathrm{~Hz}$ represents rotating frequency, $A_{i}$ denotes the amplitude modulation with the cycle of $1 / f_{r}, f_{n}=3200 \mathrm{~Hz}$ is the natural frequency of the system and $r=0.05$ is the damping coefficient. $t_{i}=0.01 T-0.02 T$ denotes the delay caused by the slippage of rolling element in $i$ th cycle. $T=1 / 185 \mathrm{~s}$ represents the period during which the fault occurs, which means the fault characteristic frequency is $185 \mathrm{~Hz}$. The harmonic $B(t)$ is applied to simulate the interference components and the value of $f_{1}$ and $f_{2}$ is $80 \mathrm{~Hz}$ and $100 \mathrm{~Hz}$ respectively. $m(t)$ represents random impulsive noise, $n(t)$ represents Gaussian noise. The signal-to-noise ratio of this signal is $-8.18 \mathrm{~dB}$ if no white noise is added, and the ratio is $-14.4 \mathrm{~dB}$ after adding white noise. The sampling frequency is $32768 \mathrm{~Hz}$ and the sampling time is $1 \mathrm{~s}$.

In order to prove that $N_{2}$ is suitable for selecting the center frequency of the filter, the bandwidth is fixed at five times the fault characteristic frequency, and the value of $N_{2}$ under different center frequencies are calculated, the relationship between center frequency and $N_{2}$ is 
shown in Fig. 1. From which we have conclusion that $N_{2}$ shows a trend of increasing first and then decreasing, and reaches the maximum when the center frequency is selected as $3200 \mathrm{~Hz}$. At the same time, when the selected center frequency is less than $3200 \mathrm{~Hz}$, as it increases, the filtered signal contains more useful information, which results in the better performance of envelope analysis; while when it is greater than $3200 \mathrm{~Hz}$, with the increasing of center frequency, the useful information contained in the filtered signal decreases, so the effect of fault feature extraction deteriorates gradually. Therefore, the relationship between $N_{2}$ and center frequency is consistent with the theoretical analysis, which indicates $N_{2}$ is suitable for selecting the center frequency.

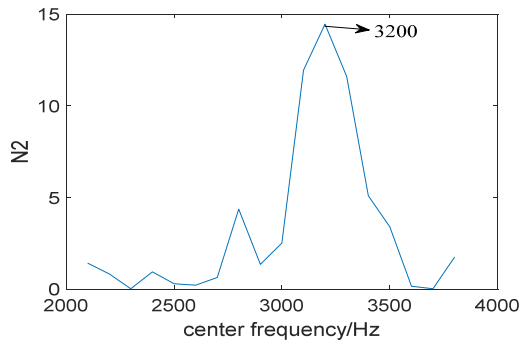

Fig. 1. Curve of relationship between center frequency and $N_{2}$

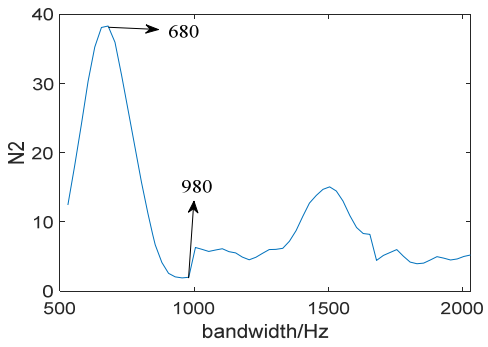

Fig. 2. The curve of the relationship between $N_{2}$ and bandwidth

To explore the relationship between bandwidth and $N_{2}$, we fix the center frequency at $3200 \mathrm{~Hz}$, and the bandwidth is selected between three times and eleven times of fault characteristic frequency, which is $[555,2035]$. The corresponding curve of them is shown in Fig. 2, Where $N_{2}$ reaches the maximum when the bandwidth is $680 \mathrm{~Hz}$, the minimum when the bandwidth is $980 \mathrm{~Hz}$.

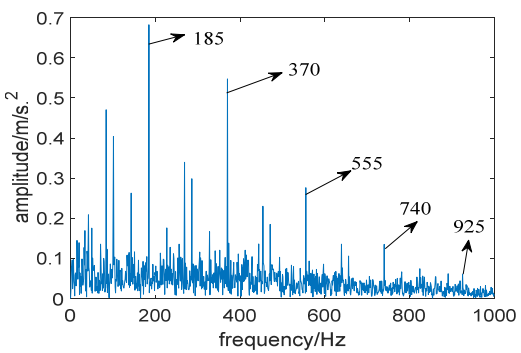

a)

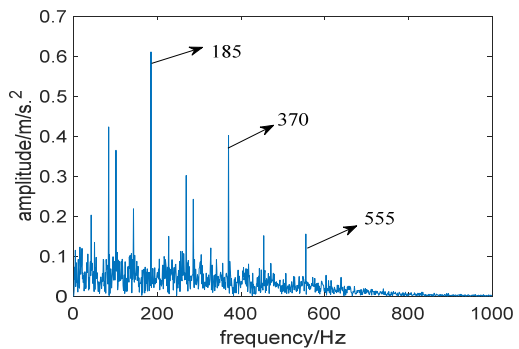

b)

Fig. 3. The squared envelope spectrum of the filtered signal at different bandwidth:

a) the bandwidth $980 \mathrm{~Hz}, \mathrm{~b}$ ) the bandwidth $680 \mathrm{~Hz}$

In order to prove that $N_{2}$ is unreliable for selecting the bandwidth, two band-pass filters with a center frequency of $3200 \mathrm{~Hz}$ and bandwidths of $980 \mathrm{~Hz}$ and $680 \mathrm{~Hz}$ are designed respectively to filter the simulation signal and perform envelope demodulation. Fig. 3(a) is the squared envelope spectrum of the filtered signal at a bandwidth of $980 \mathrm{~Hz}$, where fault characteristic frequency $185 \mathrm{~Hz}$ and its harmonics $370 \mathrm{~Hz}, 555 \mathrm{~Hz}$ and $740 \mathrm{~Hz}$ are clearly visible, and one of its harmonics $925 \mathrm{~Hz}$ can even been identified faintly. Fig. 3(b) is the squared envelope spectrum of the filtered signal when the bandwidth is $680 \mathrm{~Hz}$, where only the fault characteristic frequency and two of its harmonics $370 \mathrm{~Hz}$ and $555 \mathrm{~Hz}$ can be identified. Comparing Fig. 3(a) with Fig. 3(b), the squared envelope spectrum of the filtered signal with the bandwidth of $980 \mathrm{~Hz}$ performs better. Therefore, $N_{2}$ is not appropriate for selecting the bandwidth.

From the above analyses, we come to the conclusion that $N_{2}$ is suitable for selecting the center frequency of the filter, but is unreliable for selecting the bandwidth. Hence, in this paper, the bandwidth is fixed at five times the fault characteristic frequency, and $N_{2}$ is utilized for selecting the optimal center frequency. 


\subsubsection{Whale optimization algorithm}

Whale optimization algorithm [21] inspired by humpback whale hunting behavior is a meta-heuristic optimization algorithm, and its mathematical model can be expressed as the following three steps [21]:

1) Encircling prey.

Humpback whales can recognize the location of prey and encircle them, and this behavior can be represented by the following formulas:

$\left\{\begin{array}{l}D=\left|C X^{*}(t)-X(t)\right| \\ X(t+1)=X^{*}(t)-A D\end{array}\right.$

where $t$ indicates the current iteration, $X^{*}$ is the position vector of the best solution obtained so far, $X$ is the position vector, $|\cdot|$ is the absolute value, and 'stands for dot product. To obtain the optimal solution, the position of the whale needs to be updated according to the following formula during each iteration:

$\left\{\begin{array}{l}A=2 a r-a, \\ C=2 r\end{array}\right.$

where $a$ linearly from 2 to 0 during the iterations and $r$ is selected randomly between 0 and 1 .

2) Bubble-net attacking method.

The bubble-net attack method is a unique attack method for humpback whales. Two methods are designed to mathematically model its behavior.

The first method is shrinking encircling mechanism, which can be realized by reducing the value of $a$ in Eq. (9). The fluctuation range of $A$ is determined by the value of $a$. Here we set $A$ as random values in $[-1,1]$, then if $0 \leq A \leq 1$, whales will attack towards the prey.

The second approach is called spiral updating position, which is utilized to mimic the helix-shaped movement of whales based on the position of the whale and prey. The formula can be represented as follows:

$X(t+1)=D^{\prime} e^{b l} \cos (2 \pi l)+X^{\prime}(t)$,

where, $D^{\prime}=\left|X^{*}(t)-X(t)\right|$ denotes the distance between the $i$ th whale and its prey, $b$ is a constant utilized to simulate the shape of the logarithmic spiral, $l$ is selected randomly between -1 and 1 .

To model the bubble-net attack method, assuming that the humpback whale has a $50 \%$ probability of choosing a method to update its position, the following modeling can be performed:

$X(t+1)=\left\{\begin{array}{l}X^{*}(t)-A D, \quad 0 \leq p<0.5, \\ D^{\prime} e^{b l} \cos (2 \pi l)+X^{*}(t), \quad 0.5 \leq p \leq 1,\end{array}\right.$

where $p$ is a random number between 0 and 1 .

3) Search for prey.

The same approach based on the variation of the $A$ vector can be utilized to search for prey, if $|A| \geq 1$, the search agent will be forced to move far away from a reference whale. This allows the WOA algorithm to perform a global search. The mathematical model is as follows:

$\left\{\begin{array}{l}D=\left|C X_{\text {random }}(t)-X(t)\right| \\ X(t+1)=X_{\text {random }}(t)-A D\end{array}\right.$

where, $X_{\text {random }}$ indicates the random position vector selected from the current population.

According to the above analyses, The WOA algorithm can be illustrated as follows [15]: 
Step 1: Initialize the whales' population $X_{i}(i=1,2, \ldots, n)$.

Step 2: Initialize $a, A, C, l$ and $p$.

Step 3: Calculate the fitness of each search agent. $X^{*}$ is the best search agent;

Step 4: When $p<0.5$, if $|A|<1$, update the current population position with Eq. (8), else, update the current population position with Eq. (12); when $0.5<p<1$, use Eq. (10) to update the position of the current population;

Step 5: Update $a, A$ and $C$;

Step 6: Calculate the fitness of each search agent.

Step 7: Update the value of $X^{*}$.

Step 8: Determine whether the current iteration number $t<$ maximum number of iteration, if yes, repeat Steps 4 to 7 . Else, return to $X^{*}$.

\subsubsection{Using WOA to optimize filter center frequency}

Considering the strong robustness of $N_{2}$ and the powerful optimization ability of WOA, this paper fixes the bandwidth at five times the fault characteristic frequency, then uses WOA to quickly select the optimal center frequency $X^{*}$, and $N_{2}$ is used as the objective function of WOA.

\subsection{Cross-correlation spectrum analysis}

Due to the fact that there still contains some noise interference in the filtered signal, it is necessary to further suppress the in-band noise. Klausen [22] pointed out that cross-correlation analysis could enhance the same frequency components in two signals and suppress uncorrelated components, so as to achieve the goal of removing random noise.

Considering the advantages of enhancing the impact component of signal and easy calculation, higher order energy operator [23] is often used to extract the fault feature of rolling bearings. Therefore, based on literature [22], we propose to utilize the optimal two energy operators of the filtered signal for cross-correlation spectrum analysis. The choice of the energy operator affects the extraction of fault features, so this paper constructs FCI to select the optimal two energy operators for cross-correlation spectrum analysis, the expression is as follows:

$$
F C I=\frac{\operatorname{sum}\left(m^{2}(k * \text { fault }) * k^{2}\right)}{\operatorname{sum}\left(m^{2}(1: K * \text { fault })\right)-\operatorname{sum}\left(m^{2}(k * \text { fault })\right)}
$$

where, $m$ represents the amplitude of the squared envelope spectrum, $k$ represents the order of the fault feature, and $k=(1,2, \ldots, K), K$ takes 3 , fault represents fault feature frequency. FCI can be understood as the ratio of the energy of fault characteristic frequency in the squared envelope spectrum to the rest energy within the spectrum range. Therefore, the larger the FCI is, the higher the energy proportion corresponding to fault characteristic frequency will be, and the better the fault feature extraction effect will be. Note that the energy of each order of fault characteristic frequency is multiplied by $k^{2}$, this is because the higher order of fault characteristic frequencies are more difficult to detect, so a greater weight is given.

At the same time, for discrete signals, the higher order energy operator can be calculated as [23]:

$$
E O_{k}[x(n)]=x(n) x(n+k-2)-x(n-1) x(n+k-1),
$$

where, $k$ is the order of the energy operator. Considering that the higher order energy operator will increase the high frequency noise in the signal while enhancing the fault impact, this paper sets the maximum order to 10 .

According to FCI, the optimal two energy operators are selected and expressed as $E O_{k 1}[x(n)]$ and $E O_{k 2}[x(n)]$ respectively. Zero-average them as follows: 
$\left\{\begin{array}{l}E O_{k 1}^{Z m}[x(n)]=E O_{k 1}[x(n)]-\operatorname{mean}\left\{E O_{k 1}[x(n)]\right\} \\ E O_{k 2}^{Z m}[x(n)]=E O_{k 2}[x(n)]-\operatorname{mean}\left\{E O_{k 2}[x(n)]\right\}\end{array}\right.$

Therefore, the cross-correlation calculation and normalization of two order energy operators can be obtained:

$R_{E O_{k 1}^{Z m} E O_{k 2}^{Z m}}(m)= \begin{cases}\frac{1}{L} \sum_{n=0}^{L-m-1} E O_{k 1}^{Z m}\left[x(n+m) E \tilde{O}_{k 2}^{z m} x(n)\right], & m \geq 0, \\ \tilde{R}_{E O_{k 1}^{Z m} E O_{k 2}^{z m}}(-m), & m<0,\end{cases}$

where, $L$ represents the length of signal.

\subsection{The principle of the proposed algorithm based on conditional variance statistic and cross-correlation spectrum}

Considering that conditional variance statistic can be used as the objective function for selecting the center frequency of the filter, but not bandwidth, we choose to fix the bandwidth at five times the fault characteristic frequency, and use WOA to select the optimal center frequency quickly. To further suppress in-band noise, cross-correlation analysis based on higher order energy operator is utilized. In order to better display the proposed algorithm, the flowchart is shown in Fig. 4.

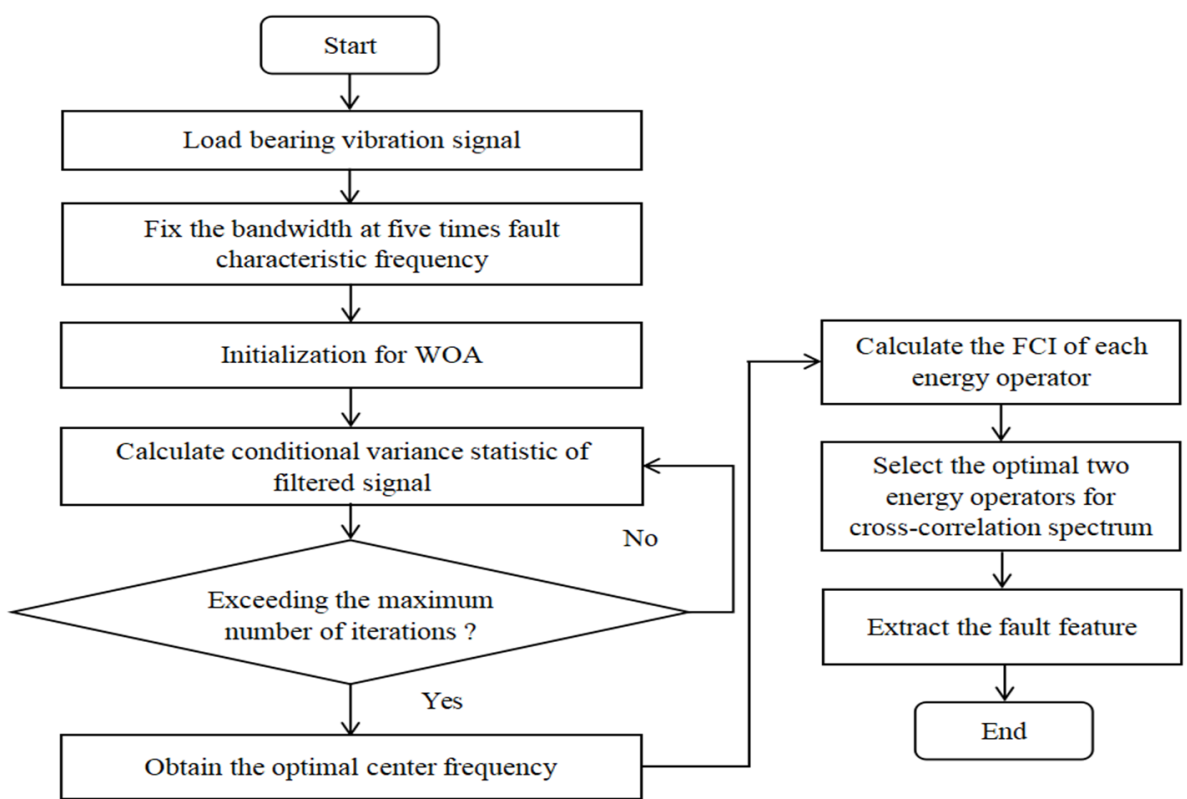

Fig. 4. The flowchart of the proposed algorithm

\section{Simulation verification}

To verify the effectiveness of the proposed algorithm for extracting the fault feature, this section constructs a simulation signal under strong background noise, where repetitive transients and harmonic components are the same as those in for Eq. (7). The signal-to-noise ratio of the signal is $-8.2 \mathrm{~dB}$ if no white noise is added, and the ratio is $-15.2 \mathrm{~dB}$ after adding white noise.

Fig. 5 are the time domain waveform of the simulation signal and its squared envelope 
spectrum. The envelope spectrum is obtained by performing Hilbert transform and FFT on the signal directly. Disturbed by strong background noise, repetitive transients related to the local damage can hardly be observed in the time domain waveform and only fault characteristic frequency $185 \mathrm{~Hz}$ along with one of its harmonics $370 \mathrm{~Hz}$ are able to be observed in the envelope spectrum.

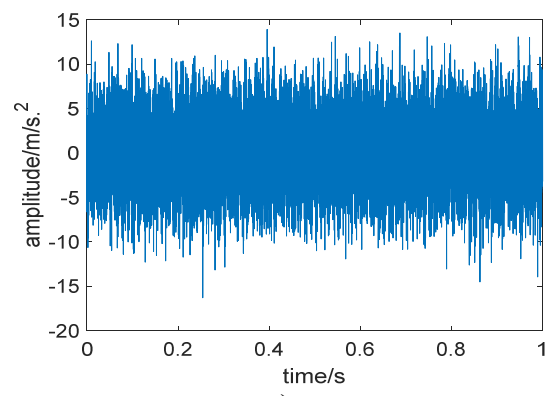

a)

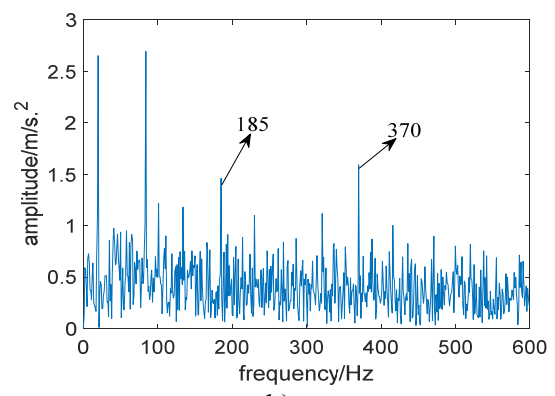

b)

Fig. 5. Simulation signal: a) time domain waveform; b) squared envelope spectrum of a)

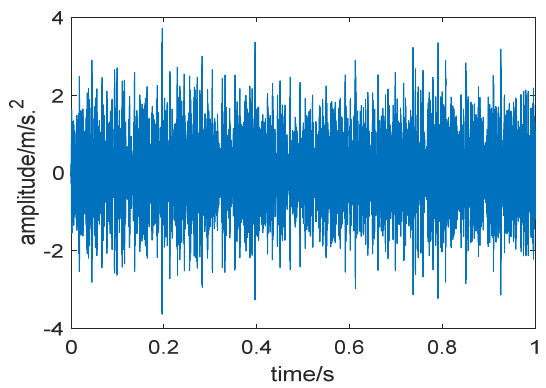

Fig. 6. Time domain waveform of the filtered signal

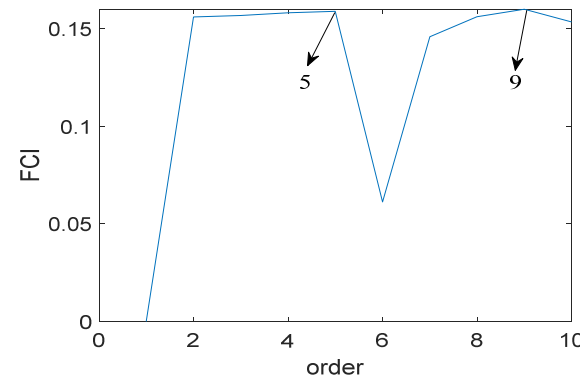

a)

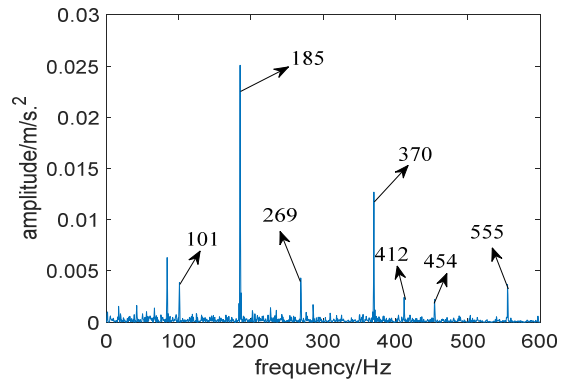

b)

Fig. 7. Results of the proposed method: a) the curve of FCI; b) cross-correlation spectrum

Next, the proposed algorithm is applied to the fault simulation signal. The fixed bandwidth is five times of fault characteristic frequency, namely $925 \mathrm{~Hz}$; the optimal center frequency selected by whale optimization algorithm is $3270 \mathrm{~Hz}$, which is basically consistent with the actual center frequency of $3200 \mathrm{~Hz}$. Fig. 6 is the time domain waveform of the filtered signal, where background noise is significantly suppressed. Further, the filtered signal is analyzed by higher order energy operator. Fig. 7(a) shows the relationship between the order of energy operator and FCI, from which the optimal two energy operators with the largest FCI are selected for cross-correlation spectrum analysis, namely the fifth and ninth order; Fig. 7(b) is the cross-correlation spectrum, where noise components are obviously suppressed, fault characteristic frequency $185 \mathrm{~Hz}$ and its harmonics $370 \mathrm{~Hz}$ and $555 \mathrm{~Hz}$ are prominent, what's more, the sidebands $101 \mathrm{~Hz}, 269 \mathrm{~Hz}, 412 \mathrm{~Hz}$ 
and $454 \mathrm{~Hz}$ which are modulated by rotating frequency can be observed too. Compared with Fig. 5(b), the proposed algorithm performs better.

In order to highlight the superiority of the proposed method, now we use Fast Kurtogram, EMD and Fast-SC [24] to deal with the simulation signal.

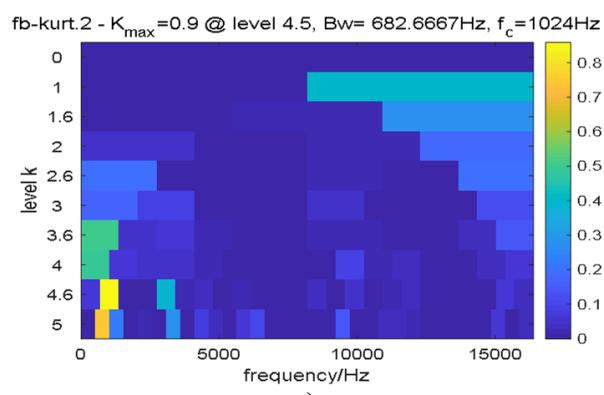

a)

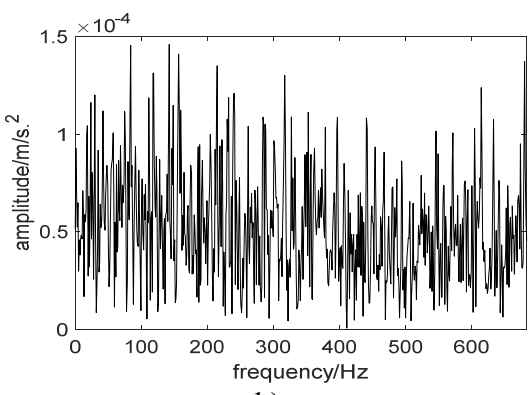

b)

Fig. 8. Results of fast kurtogram: a) the paving of fast kurtogram;

b) the squared envelope spectrum of the signal filtered by fast kurtogram

Fig. 8 are the results of Fast Kurtogram. Due to the interference of strong impulsive noise, the optimal center frequency determined by Fast Kurtogram is $1024 \mathrm{~Hz}$, which is inconsistent with the actual center frequency, and the selected bandwidth is $682.7 \mathrm{~Hz}$; the squared envelope spectrum of the selected frequency band showed in Fig. 8(b) also contains no useful information. Comparing Fig. 7(b) with Fig. 8(b), the proposed algorithm performs better.

Since the useful components in the original signal are mostly concentrated in the first few intrinsic mode functions (IMF) obtained by EMD, we only take the first four IMFs for analysis. Fig. 9(a) is the time domain waveform of the first four IMFs and Fig. 9(b) is their squared envelope spectrum, from which we come to the conclusion that the third IMF contains the most useful information. To observe the performance of EMD more intuitively, we enlarge the squared envelope spectrum of the third IMF, which is shown in Fig. 10. In Fig. 10, although fault characteristic frequency $185 \mathrm{~Hz}$ and its harmonics can be observed, there still contains some noise interference. Comparing Fig. 7(b) with Fig. 10, the proposed algorithm performs better.

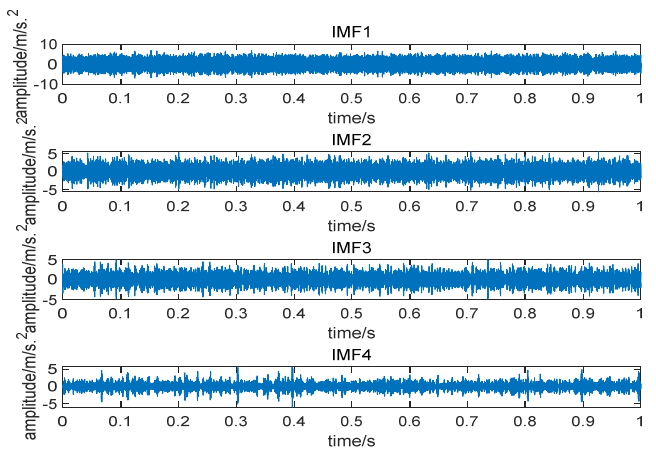

a)

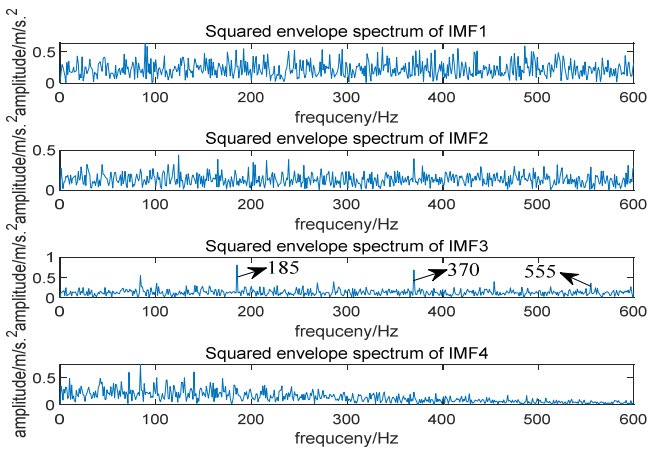

b)

Fig. 9. Results of EMD: a) the time domain waveform of the first four IMFs; b) the squared envelope spectrum of the first four IMFs

What is more, the analysis result of Fast-SC is shown in Fig. 11, where fault characteristic frequency and its harmonies can also be observed in its enhanced envelope spectrum. However, compared with Fig. 7(b), there still contains some noise interference, which indicates that the proposed algorithm performs better. 


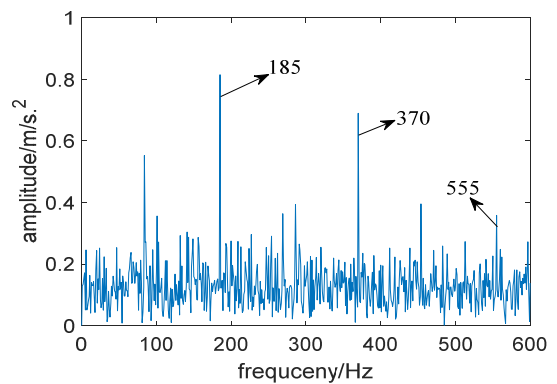

Fig. 10. The squared envelope spectrum of the third IMF

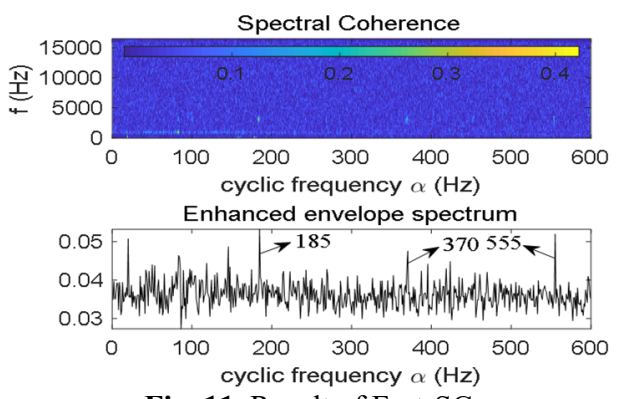

Fig. 11. Result of Fast-SC

\section{Experimental verification}

In this section, the vibration signal collected from a bearing test rig is analyzed to further evaluate the effectiveness of the proposed method for fault diagnosis of rolling element bearings. The test rig shown in Fig. 12 contains several main components, including bearing support structure, main shaft, experimental bearing, servo-driven motor, radial loading device, axial loading device and control system et al.

The experimental bearing type is $7010 \mathrm{C}$ with a local damage on the outer race generated by laser wire-electrode cutting, the fault size of the outer race was $0.5 \mathrm{~mm}$ (width) $\times 0.5 \mathrm{~mm}$ (depth). The outer diameter of the bearing is $80 \mathrm{~mm}$ and the inner diameter is $50 \mathrm{~mm}$, the rolling element diameter is $8.7 \mathrm{~mm}$, the number of rolling elements is 19 , and the contact angle is $15^{\circ}$. The test speed is $3000 \mathrm{r} / \mathrm{min}$, in order to obtain detailed signal data, the sampling frequency is set to the highest sampling frequency of the instrument, which is $131072 \mathrm{~Hz}$, and the sampling time is $1 \mathrm{~s}$. The theoretical fault characteristic frequency is calculated as $413.6 \mathrm{~Hz}$.

In order to reduce the calculation, the signal is resampled to $32768 \mathrm{~Hz}$. Fig. 13 are the time domain waveform the experimental signal and its squared envelope spectrum. Due to the interference of strong background noise, repetitive transients related to local damage can hardly be observed in the time domain waveform and its squared envelope spectrum also contains no useful information.

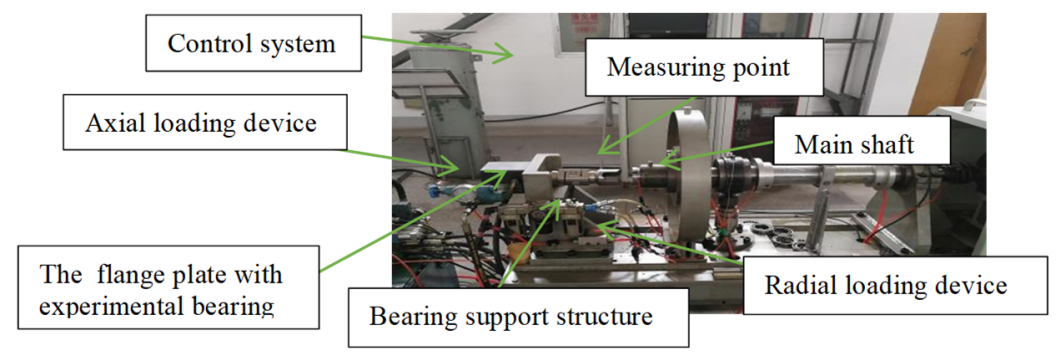

Fig. 12. The test rig

Next, the proposed algorithm is applied to the experimental signal. The bandwidth is fixed at five times fault characteristic frequency; the optimal center frequency selected by whale optimization algorithm is $2971 \mathrm{~Hz}$. Fig. 14 is the time domain waveform of the filtered signal, compared with Fig. 13(a), noise interference is effectively suppressed. Further, the filtered signal is analyzed by higher order energy operator. Fig. 15(a) reflects the relationship between the order of energy operator and FCI, and the optimal two energy operators with the largest FCI are selected for cross-correlation spectrum analysis, namely the ninth and tenth order. Fig. 15(b) is the cross-correlation spectrum, it is obviously that noise components are suppressed, fault characteristic frequency $417 \mathrm{~Hz}$ and its harmonics $833 \mathrm{~Hz}$ and $1250 \mathrm{~Hz}$ are prominent. Compared 
with Fig. 13(b), the proposed algorithm performs better. What's more, note that due to the fluctuation of the rotating speed or the sliding of the rolling bearing, the actual fault characteristic frequency is not equal to the theoretical value.

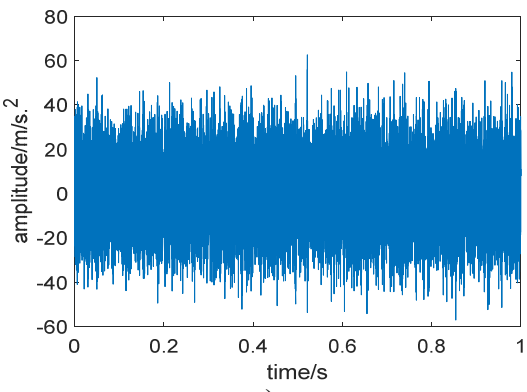

a)

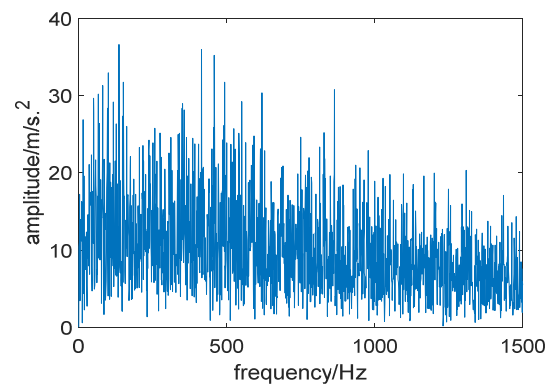

b)

Fig. 13. Experimental bearing vibration signal:

a) time domain waveform; b) squared envelope spectrum of a)

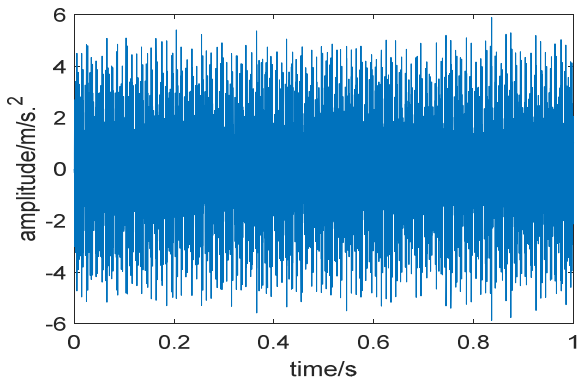

Fig. 14. Time domain waveform of the filtered signal

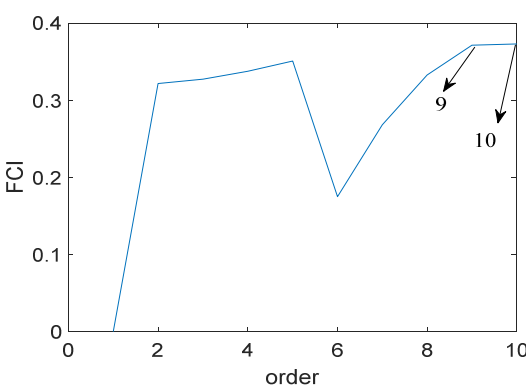

a)

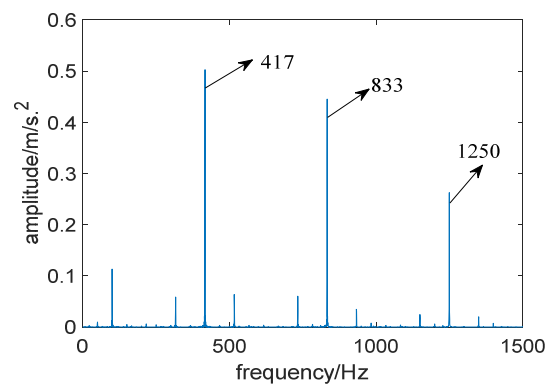

b)

Fig. 15. Results of the proposed method: a) the curve of FCI; b) cross-correlation spectrum

To further prove that $N_{2}$ is not suitable for selecting the bandwidth of the filter, we fix the center frequency at $2971 \mathrm{~Hz}$, and the bandwidth is selected between three and eleven times of fault characteristic frequency. The relationship between $N_{2}$ and bandwidth is shown in Fig. 16, where $N_{2}$ reaches the maximum when the bandwidth is $2000 \mathrm{~Hz}$, and the minimum when the bandwidth is $3100 \mathrm{~Hz}$. Then two band-pass filters with a center frequency of $2971 \mathrm{~Hz}$ and bandwidths of $2000 \mathrm{~Hz}$ and $3100 \mathrm{~Hz}$ are designed to filter the experimental signal and perform envelope demodulation. Fig. 17(a) and Fig. 17(b) are the squared envelope spectrum of the filtered signal when the bandwidth is $2000 \mathrm{~Hz}$ and $3100 \mathrm{~Hz}$, comparing Fig. 17(a) with Fig. 17(b), we find both of them perform well. Therefore, it is unreliable to select the bandwidth by $\mathrm{N}_{2}$.

For comparison, Fast Kurtogram, EMD and Fast-SC are now utilized to analyze the experimental signal. 


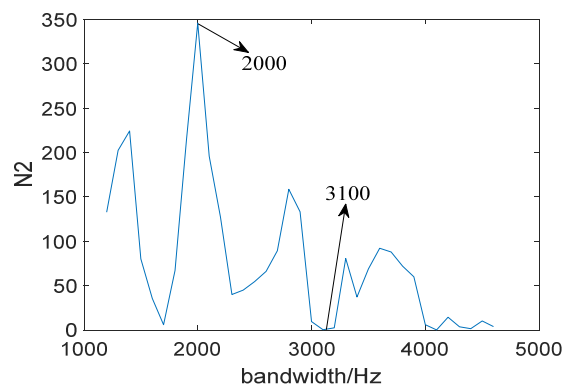

Fig. 16. The curve of the relationship between $N_{2}$ and bandwidth

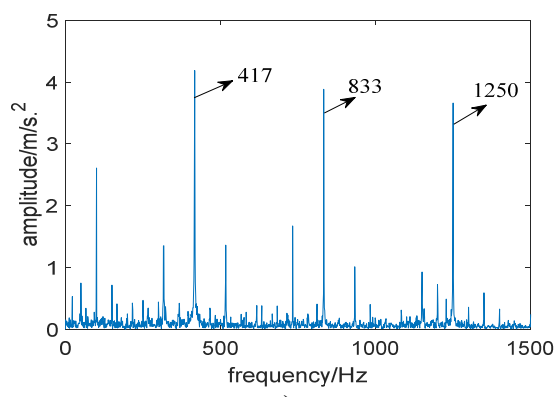

a)

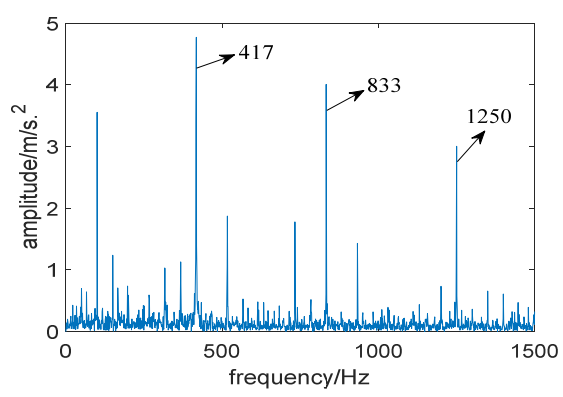

b)

Fig. 17. The squared envelope spectrum of the filtered signal at different bandwidth:

a) the bandwidth $2000 \mathrm{~Hz}$, b) the bandwidth $3100 \mathrm{~Hz}$

Fig. 18 are the results of Fast Kurtogram. Due to the interference of strong background noise, optimal center frequency determined by Fast Kurtogram is $256 \mathrm{~Hz}$, and the selected bandwidth is $512 \mathrm{~Hz}$; in the squared envelope spectrum of the optimal analysis frequency band, the prominent frequency $50 \mathrm{~Hz}$ corresponds to the rotation frequency of the rolling bearing, however, fault characteristic frequency and its harmonics cannot be Identified, which indicates Fast Kurtogram fails to extract the fault feature.

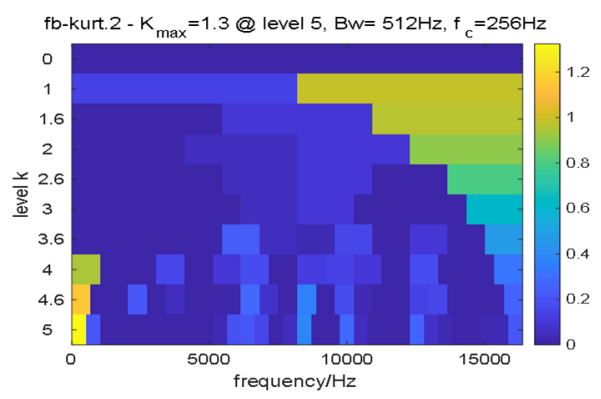

a)

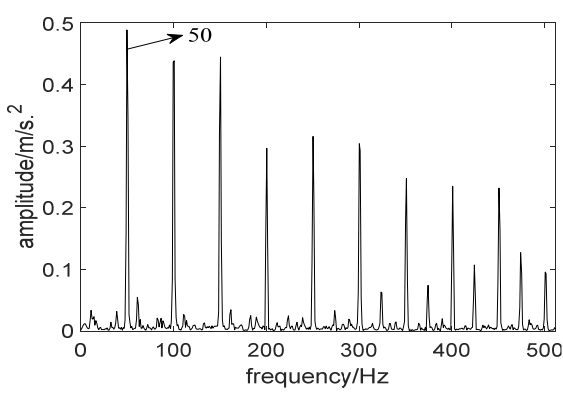

b)

Fig. 18. Results of fast kurtogram: a) the paving of fast kurtogram;

b) the squared envelope spectrum of the signal filtered by fast kurtogram

Similar to the simulation signal, we only give the time domain waveform and their squared envelope spectrum of the first four IMFs obtained by EMD, which is shown in Fig. 19, and the squared envelope spectrum of the third IMF which contains the most useful information is shown in Fig. 20. In Fig. 20, although fault characteristic frequency $417 \mathrm{~Hz}$ and its harmonics can be observed, there still exists some noise interference, compared with Fig. 15(b), the proposed algorithm performs better.

What's more, the analysis result of Fast-SC is shown in Fig. 21, where fault characteristic frequency and its harmonies are also prominent. However, compared with Fig. 15(b), there still 
exists some noise interference in the enhanced envelope spectrum, so the proposed algorithm performs better.

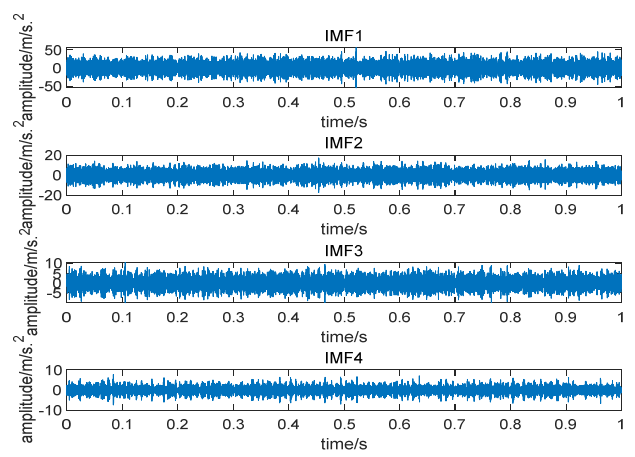

a)

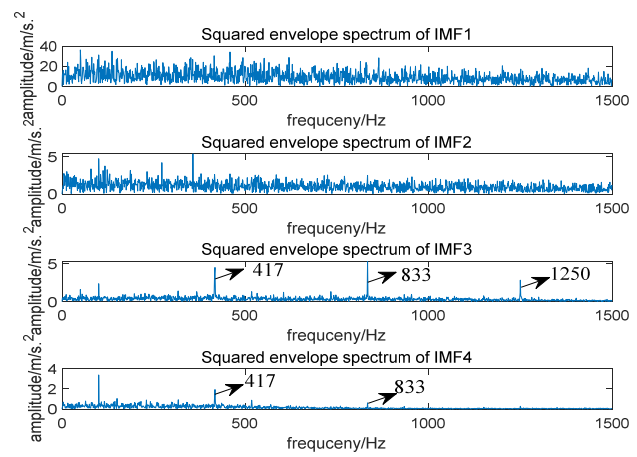

b)

Fig. 19. Results of EMD: a) the time domain waveform of the first four IMFs;

b) the squared envelope spectrum of the first four IMFs

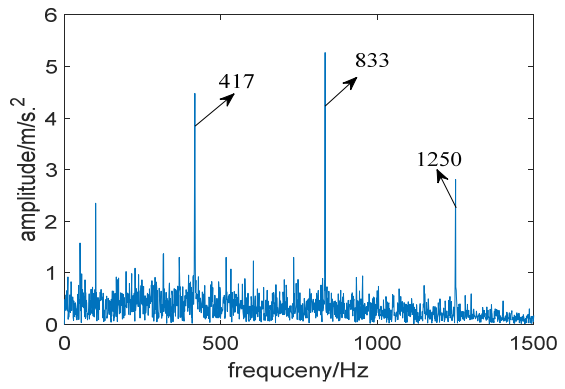

Fig. 20. The squared envelope spectrum of the third IMF

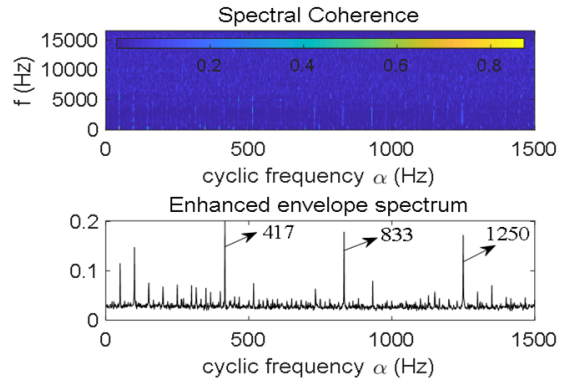

Fig. 21. Result of Fast-SC

\section{Conclusions}

From the analyses of simulation and experimental signal, the algorithm proposed in this paper based on conditional variance statistic and cross-correlation spectrum analysis is feasible, and we have the conclusions as follow:

1) Compared with kurtosis and Gini index, conditional variance statistic is more robust and can be utilized as the objective function for selecting the optimal center frequency. However, we find that conditional variance statistic is undesirable for selecting the optimal bandwidth.

2) Finding the optimal center frequency by iteration will be affected by step size. If the step size is too long, the accurate center frequency cannot be selected; if the step size is too short, the calculation efficiency will be affected. In this paper, the bandwidth is fixed at five times fault characteristic frequency and WOA is applied to quickly select the optimal center frequency, what is more, $N_{2}$ is utilized as the objective function, so both of the calculation efficiency and accuracy of the selection of center frequency can be taken into consideration. To further suppress the noise interference, cross-correlation spectrum analysis is applied.

3) The analyses of the simulation and experimental signal indicate that the proposed method can effectively extract the fault feature of rolling bearings under strong background noise. Compared with classic Fast Kurtogram, EMD and Fast-SC, the proposed algorithm performs better. 


\section{References}

[1] Gu Xiao-hui, Yang Shao-pu, Li Yong-qiang, et al. Multi-objective informative frequency band selection based on negentropy-induced grey wolf optimizer for fault diagnosis of rolling element bearings. Sensors, Vol. 20, Issue 7, 2020, p. 1845.

[2] Xiong Qing, Xu Yan-hai, Peng Yi-qiang Low-speed rolling bearing fault diagnosis based on EMD denoising and parameter estimate with alpha stable distribution. Journal of Mechanical Science and Technology, Vol. 31, Issue 4, 2017, p. 1587-1601.

[3] Yan Xiao-an, Jia Min-ping Application of CSA-VMD and optimal scale morphological slice bispectrum in enhancing outer race fault detection of rolling element bearings. Mechanical Systems and Signal Processing, Vol. 122, 2019, p. 56-86.

[4] Cheng Y., Zhou N., Zhang W., et al. Application of an improved minimum entropy deconvolution method for railway rolling element bearing fault diagnosis. Journal of Sound and Vibration, Vol. 425, 2018, p. 53-69.

[5] Medonald G. L., Qing Zhao, Ming Zuo J. Maximum correlated kurtosis deconvolution and application on gear tooth chip fault detection. Mechanical Systems and Signal Processing, Vol. 33, 2012, p. 237-255.

[6] Zhou Cheng-Jiang, Ma Jun, Wu Jian-de, et al. A parameter adaptive MOMEDA method based on grasshopper optimization algorithm to extract fault features. Mathematical Problems in Engineering, Vol. 2019, 2019, p. 718253.

[7] Marco Buzzoni, Jérôme Antoni, Gianluca D'Elia Blind deconvolution based on cyclostationarity maximization and its application to fault identification. Journal of Sound and Vibration, Vol. 432, 2018, p. 569-601.

[8] Randall R. B., Antoni J. Rolling element bearing diagnostics - a tutorial. Mechanical Systems and Signal Processing, Vol. 25, Issue 2, 2011, p. 485-520.

[9] Antoni J. Fast computation of the kurtogram for the detection of transient faults. Mechanical Systems and Signal Processing, Vol. 21, Issue 1, 2007, p. 108-124.

[10] Abboud D., Elbadaoui M., Smith W. A., et al. Advanced bearing diagnostics: A comparative study of two powerful approaches. Mechanical Systems and Signal Processing, Vol. 114, 2019, p. 604-627.

[11] Xu Yong-gang, Tian Wei-kang, Zhang Kun Application of an enhanced fast kurtogram based on empirical wavelet transform for bearing fault diagnosis. Measurement Science and Technology, Vol. 30, Issue 3, 2019, p. 035001.

[12] Barszcz T., Jabłoński A. A novel method for the optimal band selection for vibration signal demodulation and comparison with the Kurtogram. Mechanical Systems and Signal Processing, Vol. 25, Issue 1, 2011, p. 431-451.

[13] Antoni J. The infogram: Entropic evidence of the signature of repetitive transients. Mechanical Systems and Signal Processing, Vol. 74, 2016, p. 73-94.

[14] Justyna Hebda-Sobkowicza, Radosław Zimroza, Marcin Piterab Informative frequency band selection in the presence of non-Gaussian noise - a novel approach based on the conditional variance statistic with application to bearing fault diagnosis. Mechanical Systems and Signal Processing, Vol. 145, 2020, p. 106971.

[15] Miao Yong-hao, Zhao Ming, Lin Jing Improvement of kurtosis-guided-grams via Gini index for bearing fault feature identification. Measurement Science and Technology, Vol. 28, Issue 12, 2017, p. 125001.

[16] Miao Yong-hao, Zhao Ming, Hua Jia-dong. Research on sparsity indexes for fault diagnosis of rotating machinery. Measurement, Vol. 158, 2020, p. 107733.

[17] Hebda-Sobkowicz Justyna, Zimroz Radosław, Wyłomanska Agnieszka Selection of the informative frequency band in a bearing fault diagnosis in the presence of non-gaussian noise-comparison of recently developed methods. Applied Sciences, Vol. 10, 2020, p. 2657.

[18] Duan Jie, Shi Tie-lin A narrowband envelope spectra fusion method for fault diagnosis of rolling element bearings. Measurement Science and Technology, Vol. 29, Issue 12, 2018, p. 125106.

[19] Jaworski P., Pitera M. The 20-60-20 rule. Discrete and Continuous Dynamical Systems - Series B, Vol. 21, Issue 4, 2016, p. 1149-1166.

[20] Jelito D., Pitera M. New fat-tail normality test based on conditional second moments with applications to finance. arXiv:1811.05464, 2018.

[21] Mirjalili S., Lewis A. The whale optimization algorithm. Advances in Engineering Software, Vol. 95, 2016, p. 51-67. 
[22] Klausen A., Robbersmyr K. G. Cross-correlation of whitened vibration signals for low-speed bearing diagnostics. Mechanical Systems and Signal Processing, Vol. 118, 2019, p. 226-244.

[23] Faghidi H., Liang M. Bearing fault identification by higher order energy operator fusion: A non-resonance based approach. Journal of sound and Vibration, Vol. 381, 2016, p. 83-100.

[24] Antoni J., Ge Xin, Hamzaoui N. Fast computation of the spectral correlation. Mechanical Systems and Signal Processing, Vol. 92, 2017, p. 248-277.

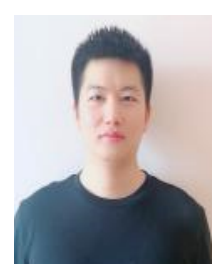

Baoyu Huang received B.E. degree in Power Engineering Institute from Naval University of Engineering, Wuhan, China, in 2018. He is now pursuing Ph.D. degree at Naval University of Engineering. His current research interest is fault diagnosis.

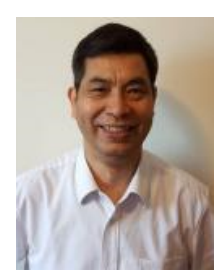

Yongxiang Zhang received the B.E. degree in Huazhong University of Science and Technology and the M.E. degree in Naval University of Engineering. He is the Professor in Naval University of Engineering at present. His current research interests including signal processing and fault diagnosis.

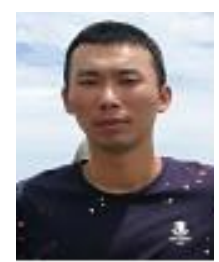

Danchen Zhu received Ph.D. degree in Power Engineering Institute from Naval University of Engineering, Wuhan, China, in 2019. Now he works at Naval Petty Officer Academy. His research interests is fault diagnosis. 